\title{
Reliability of OHM 3000 Plantar Pressure System for Measure- ment of Plantar Pressures in Healthy Indian Population
}

\author{
Neha SAWANT ${ }^{1}$, Sanket VAIDYA ${ }^{2}$.
}

${ }^{{ }_{1}}$ Research Associate, Department of Research, Kan Innovations Pvt Ltd, Mumbai, Maharashtra, India. https://orcid.org/0000-0003-3492-9865

${ }^{2}$ BPT, Department of Research, Kan Innovations Pvt Ltd, Mumbai, Maharashtra, India.

https://orcid.org/0000-0002-2674-4564

\section{ABSTRACT}

\begin{abstract}
Purpose: The aim of this study is to determine intra and inter-session repeatability and reliability of OHM 3000 for reporting static and dynamic pressures in healthy Indian population.

Methods: Using OHM 3000, twenty-eight healthy adults who did not present any gait abnormalities or musculoskeletal conditions were assessed one week apart for static and dynamic pressure. Bipedal stance test was used for assessing static variables- maximum pressure, average pressure, contact area, and weight distribution; whereas dynamic test was used for assessing dynamic variables- maximum pressure, average pressure, and contact area. Each participant performed three trials under supervision of each investigator.

Results: Reliability was devised using ICC values and coefficient of variance for static and dynamic variables. ICC values for static and dynamic variables were reported to range between 0.88 to 0.94 and 0.61 to 0.98 respectively. Covariance values were reported to be lower than $13 \%$ in static and dynamic pressures recorded by both investigators.

Conclusion: The study reports that OHM 3000 is a reliable device for assessing static and dynamic pressure in healthy adults demonstrating moderate to good reliability.

KEYWORDS: Gait, Foot, Plantar Pressure.
\end{abstract}

Address for correspondence: Dr. Neha SAWANT (PT), Research Associate, Department of Research, Kan Innovations Pvt Ltd, Mumbai, Maharashtra, India.

E-Mail: nehas@kaninnovations.com; neha8188@gmail.com

Access this Article online Quick Response code

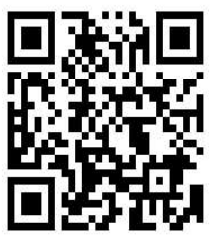

DOI: $10.16965 /$ ijpr.2021.210

Journal Information

International Journal of Physiotherapy and Research ISSN (E) 2321-1822 | ISSN (P) 2321-8975

https://www.ijmhr.org/ijpr.html

DOI-Prefix: https://dx.doi.org/10.16965/ijpr

(cc) $\mathrm{Br}+\mathrm{kit}-\mathrm{sh}$

Article Information

Received: 23 Nov 2021

Peer Review: 24 Nov 2021

Revised: None
Accepted: 29 Dec 2021

Published (O): 11 Feb 2022

Published (P): 11 Feb 2022

\section{INTRODUCTION}

Foot is an intricate and strong mechanical structure, composed of 26 bones and 33 joints secured together by multiple layers of muscles and ligaments $[1,2]$. Foot forms first point of contact between body and ground while performing activities of daily living (ADL's) like walking, running, jumping, stairclimbing $[3,4]$. In static functional activities like standing, it serves as a stable unit, while in dynamic activities like walking it acts as a lever which is essential for forward propulsion of body [5].
Foot is exposed to a tremendous amount of undue stress during these ADLs accounting for $9 \%$ to $30 \%$ of foot-related pain according to a recent literature [6]. Repetitive stress on plantar surface of foot caused during these functional activities leads to tissue deformation and injury resulting in pain, swelling, weight asymmetries, and postural changes in proximal joint structures due to biomechanical linkage.

When foot comes in contact with the ground, 
an equal magnitude force in opposite direction is exerted by ground on foot which is known as ground reaction force (GRF) [7-9].

The point of application of GRF on plantar surface of foot is called center of pressure (COP) [10]. In standing, an individual experiences GRF which is equal in magnitude to bodyweight allowing each foot to bear $50 \%$ of total body weight. While walking, this force is 3-6 times higher in comparison to standing [11]. Ambulation involves rhythmical or cyclic movement of lower limbs, with $22 \%$ of stance time spent on a single limb. Moreover, it involves forward progression of body with different parts of foot coming in contact with ground at different phases of stance cycle, leading to constant change in location and contact area of plantar surface of the loading foot. As a result, heel and forefoot bear immense amounts of pressure, highest being reported on first metatarsal head and hallux [12].

Elevated pressure on plantar surface of feet is reported as a causative factor for musculoskeletal pain in ankle, knee, hip, back, and shoulder [13]. According to Choi et al prevalence of musculoskeletal problems in adults above 18 years, accounts for $19.7 \%{ }^{(14)}$. An increase in pressure on plantar surface is often associated with a high prevalence of soft tissue injury, ulceration, stress fractures, and development of calluses [10]. Estimating factors which are responsible for causing these musculoskeletal disorders reduces burden of disability and helps in implementing patient-specific treatment strategies for better prognosis. Pressure analysis provides vital information regarding etiology of pain and related structural changes in lower limb when correlated with assessed clinical findings [15]. Other treatment options like footwear modification ${ }^{(16)}$, redistributive insoles [17], orthotic prescription, and their efficacy can be determined using pedobarographic results [10].

In recent years, plantar pressure (PP) devices have been proved to be a reliable tool in detecting pressure loading on foot [18]. Various technologically advanced devices that detect PP are available commercially, to determine pressure changes in static as well as dynamic conditions. OHM 3000 is a widely used pedobarographic device in clinical and research settings but no published studies are reported to date. To best of our knowledge, this is the first study to determine the reliability of a novel device in Indian population using OHM 3000. Owing to extensive use of this device by clinicians and researchers it is essential to establish reliability of the device. Therefore, this study aims to determine intrasession and inter-session repeatability and reliability of OHM 3000 system using test-retest analysis for reporting static and dynamic pressures in healthy Indian adults in barefoot conditions.

\section{METHODS}

Participants: A convenience sample of 28 participants $(n=28): 21$ males and 07 females, aged between 18 and 40 years of age were recruited at Research Department at Kan Innovations on voluntary basis. Demographic details of participants are mentioned in Table 1. The included participants did not suffer from any musculoskeletal, neurological disorders, or any other dysfunctions. Before commencing the study, a thorough explanation and demonstration of study procedure in bestunderstood language was given to every volunteer and informed written consent was obtained. Baseline data was collected before starting the study. Foot Posture Index [19]' (FPI-6) reported as a valid indicator to determine foot type was applied by one of the investigators at baseline to screen foot posture type exhibited by every participant, whether pronated, normal, or supinated.

Measurement Device: OHM 3000 plantar pressure system (Kan Innovations, Mumbai, India) was used to record PP while barefoot using Bipedal stance test and Dynamic test for recording static plantar pressure (SPP) and dynamic plantar pressure (DPP) respectively. This novel device is a wireless and portable device with a sensor area of $701 \times 508 \times 8 \mathrm{~mm}$, comprising of 2916 piezoresistive sensors, a sensor density of $2 / \mathrm{cm}^{2}$, a sampling frequency of $50 \mathrm{Hertz}(\mathrm{Hz})$, and a pressure range of 7 to 1034 kilopascals $(\mathrm{kPa})$. This pressure platform is calibrated using bladder system for adequate loading of each sensor uniformly, forest ablishing accurate pressure recordings [20]. 
Procedure: Sample collection was performed at research department of Kan Innovations, India. The study protocol was reviewed and ethically approved by Institutional Ethics Committee (IEC) of Kan Innovations, Mumbai, India (2020/KIPL/07/01). Device was placed at a fixed allotted position on a firm level surface for data acquisition during entire study duration. All readings were collected in barefoot conditions with sessions spaced one week apart.

Static Pressure Analysis: For measuring static pressure, participants were asked to stand in center of the mat. The participants were encouraged to stand in a natural and comfortable position with hands rolled out and positioned on either side of their body. During the procedure, participants were instructed to concentrate on a marker placed on the wall at eye level of the participant at a distance of 3 feet from the mat. They were instructed to hold this position for 20 seconds. The procedure was repeated three times for recording three trials. Readings were recorded for every participant by a primary investigator following which a rest period of 60 minutes was permitted to every participant. Then the protocol was repeated by secondary investigator. If the participant seemed to be uncomfortable or conscious while standing over the mat that reading was discarded and trial was repeated. We recorded maximum pressure (MP) in $\mathrm{kPa}$, average pressure (AP) in $\mathrm{kPa}$, weight distribution (WD) in percentage, and contact area (CA) in square centimeters $\left(\mathrm{cm}^{2}\right)$ for static pressure analysis. This baseline process was repeated after 7 days for each participant. Three trials were obtained for recording data, as it has been considered adequate for establishing the reliability of plantar pressure [21,22].

Dynamic Pressure Analysis: DPP was measured using two-step gait initiation protocol as it reports similar re-test reliability to midgait protocol and requires lesser trials ${ }^{(22,23)}$. The two-step method involved instructing the participant to stand in front of sensor matrix and to walk forward, following which, second step which strikes the mat was recorded.During the procedure, participants were instructed to walk at a natural pace without looking at the ground. Participants were asked to familiarise themselves with walking pattern over platform to ensure natural gait pattern before recording trials. Three trials of left foot followed by three trials of right foot were recorded. After a rest period of 60 minutes, procedure was repeated by secondary investigator. If participant exhibited any signs of unnatural gait pattern to achieve full contact with device, halted on mat whilst walking or targeted the mat then that trial was discarded and participant was asked to repeat the trial. We recorded MP in $\mathrm{kPa}, \mathrm{AP}$ in $\mathrm{kPa}$, and $\mathrm{CA}$ in $\mathrm{cm}^{2}$ for recording DPP measurements in this study. PP measurements were measured at baseline and repeated after 7 days by both investigators following same procedure to ensure consistency of gait characteristics of each participant.

Statistical Analysis: The data was analysed using IBM SPSS version 26 for Windows (SPSS Inc, Chicago, USA). Normality and homogeneity were measured to check whether data met parametric assumptions using KolmogoroffSmirnoff test. The standard deviation (SD) and mean for every parameter of bipedal stance test and dynamic test were calculated. Intersession reliability was calculated to determine interclass correlation coefficients (ICCS) using average of three clinical trials in static bipedal as well as dynamic parameters using $[1, \mathrm{k}]$ model. The interclass correlation coefficient (ICC) was calculated to determine intrasession reliability using $[1,1]$ and $[1, k]$ model and coefficient of variance (CoV), since these are reported to be most acceptable methods of measuring reliability of device by Atkinson and Nevill ${ }^{(24,25)}$. Interpretation of ICC values was documented in accordance with Portney and Watkins (26). CoVs determine relative variability, providing insight regarding between-trial variability which is expressed in percentage, thus enabling comparison of one data series with another.

\section{RESULTS}

A total of 29 participants participated in study, out of which 1 participant could not attend second testing session and was thus considered as a dropout. 
Table 1: Demographic Details of Participants in Study.

\begin{tabular}{lc}
\hline & $\begin{array}{c}\text { Participants } \\
\text { Mean } \pm \text { SD }\end{array}$ \\
\hline Age (in years) & $26.35 \pm 3.98$ \\
\hline Height (in cm) & $167.0 \pm 6.79$ \\
\hline Weight (in kgs) & $65.04 \pm 9.34$ \\
\hline BMI & $23.32 \pm 3.20$ \\
\hline Gender (n) & \\
Male & 21 \\
Female & 7 \\
Total & 28 \\
\hline Dominance (n) & \\
Right & 25 \\
Left & 3 \\
\hline Foot Posture Index & $4.25 \pm 1.04$ \\
\hline
\end{tabular}

Therefore, statistical analyses were carried out on 28 participants and overall mean age of study group was $26.35 \pm 3.98$ years (range 21 to 40 years) and mean BMI was $23.32 \pm 3.20 \mathrm{~kg} /$ $\mathrm{m}^{2}$. Sample size comprised of $75 \%$ male participants $(n=22)$ and mean FPI- 6 score was reported $4.25 \pm 1.04$ exhibiting a relatively normal foot type. Characteristics of participants are mentioned in Table 1.

Intrasession Reliability: The reliability (ICC and CoV) for bipedal stance test and dynamic test variables are mentioned in Table 2 and 3 respectively. In first session, ICC for bipedal stance test (Table 2 ), reliability ranged from 0.83 to 0.98 , average reliability trial was above 0.84 , and covariance ranged from $3 \%$ to $13 \%$. The ICC reliability for second session ranged from 0.84 to 0.98 , average trial reliability was above 0.91 , and covariance ranged from $2 \%$ to $12 \%$.

The ICC for intrasession reliability for dynamic test variables (Table 3 ) for first session was 0.65 to 0.96 , average trial reliability above 0.81 , and covariance ranged between $2 \%$ to $13 \%$. Whereas, ICC value for second session was 0.76 to 0.98 , average reliability above 0.91 , and covariance ranged between $2 \%$ to $11 \%$.

Table 2: Intrasession Repeatability of Static Variables.

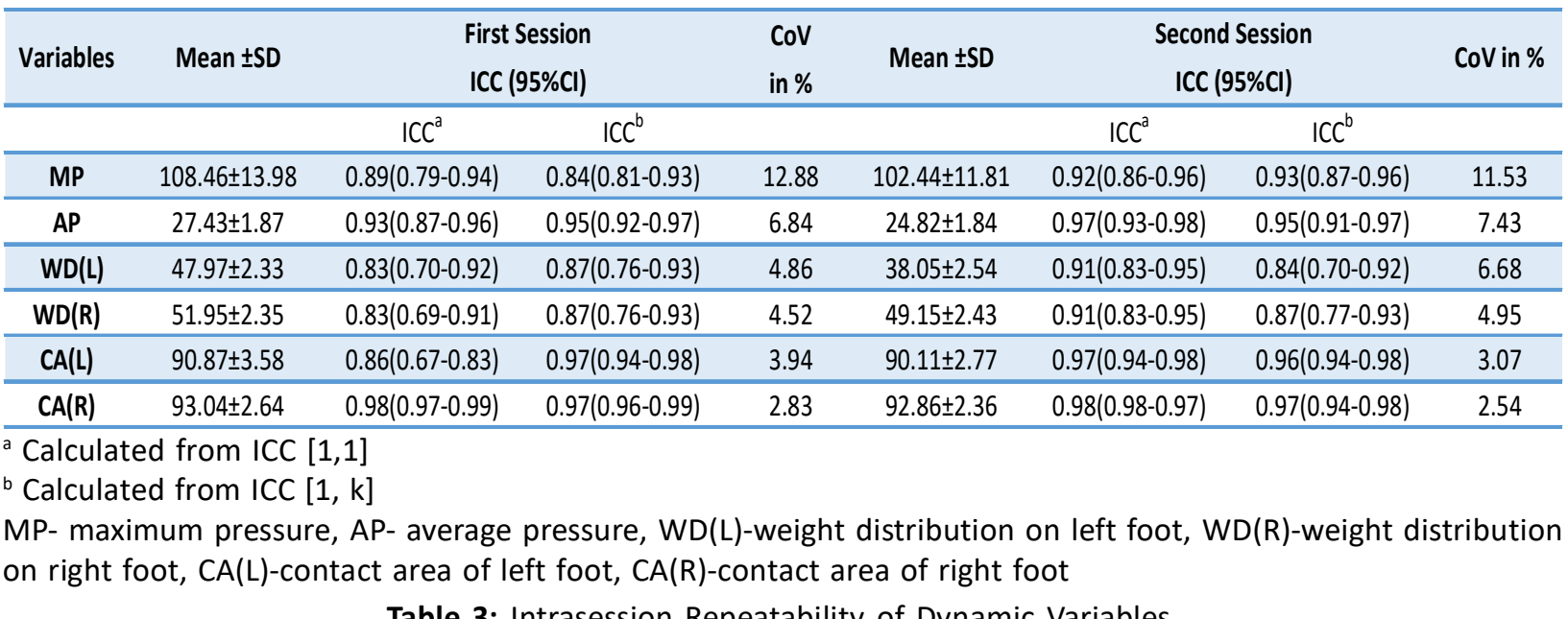

Table 3: Intrasession Repeatability of Dynamic Variables.

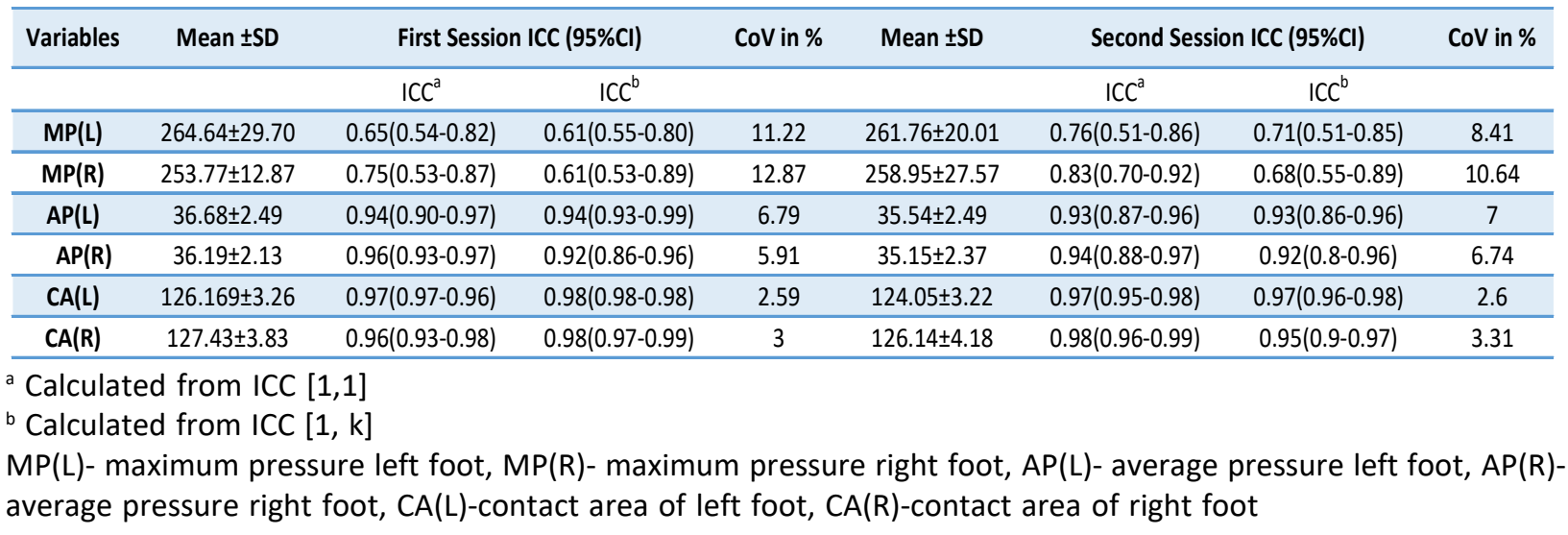

Inter-Session Reliability: For bipedal stance test ICC ranged from 0.61 to 0.98 . Overall, results which measured static variables, ICC ranged for static and dynamic recorded variables from 0.88 to 0.94 . For dynamic test variables, yielded moderate to good ICC values. 
Table 4: Intersession Reliability of Static and Dynamic Variables.Neha SAWANT, Sanket VAIDYA. Reliability of

\begin{tabular}{|c|c|c|c|}
\hline Test & Parameters & Overall Mean \pm SD & ICC $(95 \% \mathrm{Cl})$ \\
\hline \multicolumn{4}{|c|}{ of Plantar PresSures in Hea } \\
\hline \multirow{5}{*}{ Static } & AP & $26.13 \pm 1.86$ & 0.94 \\
\hline & $\mathrm{WD}(\mathrm{L})$ & $43.01 \pm 2.43$ & 0.87 \\
\hline & $W D(R)$ & $50.55 \pm 2.39$ & 0.87 \\
\hline & $\mathrm{CA}(\mathrm{L})$ & $90.49 \pm 3.18$ & 0.9 \\
\hline & $\mathrm{CA}(\mathrm{R})$ & $92.95 \pm 2.50$ & 0.92 \\
\hline \multirow{6}{*}{ Dynamic } & $\mathrm{MP}(\mathrm{L})$ & $263.20 \pm 25.86$ & 0.61 \\
\hline & $M P(R)$ & $256.36 \pm 30.11$ & 0.61 \\
\hline & $\mathrm{AP}(\mathrm{L})$ & $36.11 \pm 2.49$ & 0.94 \\
\hline & $\mathrm{AP}(\mathrm{R})$ & $35.67 \pm 2.25$ & 0.92 \\
\hline & $\mathrm{CA}(\mathrm{L})$ & $125.10 \pm 3.24$ & 0.98 \\
\hline & $C A(R)$ & $126.79 \pm 4.00$ & 0.98 \\
\hline
\end{tabular}

MP- maximum pressure, AP- average pressure, $W D(L)$-weight distribution on left foot, $W D(R)$ - weight distribution on right foot, $C A(L)$-contact area of left foot, $\mathrm{CA}(\mathrm{R})$-contact area of right foot, ICC $(95 \% \mathrm{Cl})$ - Interclass coefficient $95 \%$ confidence interval

\section{DISCUSSION}

The aim of this study was to establish repeatability and reliability of device in normal healthy population. PP analysis conducted in static and dynamic conditions provides significant clinical information indicating that areas under high pressure are likely to undergo potential damage and are prone to corns, calluses, soft tissue injury and ulceration. High-pressure area information elicited after performing pedobarography can be used as an integral part in treatment planning for various conditions like rheumatoid arthritis, osteoarthritis, and diabetic neuropathy. Hence, need for establishing accuracy and reproducibility of PP measures obtained using OHM 3000 device in normal individuals to measure foot function.

The study resulted in high intrasession and intersession reliability and repeatability of PP values using calibrated OHM 3000 platform. Intra-session repeatability was analysed for pressure parameters by calculating co-efficient of variances (CoVs) between three trials recorded during testing. Intra-session CoVs obtained for variables studied ranged between $8 \%$ to $13 \%$ and $5 \%$ to $7 \%$ for MP and AP respectively in dynamic testing. The CoVs reported in present study are relatively higher which demonstrate lower ICC values for MP in dynamic conditions with values of 0.65 for first session, 0.76 for second session which can be speculated to unavoidable and natural physiological gait variations leading to increased variability in foot loading pattern, changes in muscular activity, and body position during each trial. The inter-session and intrasession reliability of OHM 3000 device was moderate to excellent in static as well as dynamic variables.

Many studies have shown similar results in intrasession as well as intersession reliability of PP platforms in healthy individuals as well as in adults with pathologies. Our study reports ICC higher than 0.92 and CoV lower than $7 \%$ except for MP values (ICC >0.61) in dynamic state owing to gait variations.

Our results are in agreement with a study performed by Gurney et al which assessed intrasession repeatability in healthy adults using a between-day study protocol conducted on 9 asymptomatic participants for measuring MP, maximum force, and contact time using Emed platform. The intersession ICC results for areas on average were higher than 0.8 in comparison to our (ICC >0.92) which indicates good repeatability for PP variables [27].

Another study by Zammit et al was performed on 30 participants using TekScan pressure platform to measure DPP in barefoot conditions. The ICC for MP for an average of areas was higher than 0.58 and $\mathrm{CoV}$ was lower than $22 \%$ in comparison to our ICC higher than 0.61 and CoV of lower than $13 \%{ }^{(9)}$. Martineznova et al performed a study on 30 healthy adults to determine reliability and repeatability of dynamic variables. The data analysis obtained CoV lower than 7\% and ICC values higher than 0.8 for MP and AP in intrasession measurements for both variables [28].

However, it studied shoe-foot interaction using BioFoot in-shoe system in contrast to our system which studied floor-foot interaction.

The present study has demonstrated moderate to excellent reliability in normal individuals within age group of 18-40 years, however, it must be acknowledged that the results are applicable to the instrumentation used in this study and cannot be generally transferred 
to another pressure device due to different sensor technology. There are few limitations to this study that need to be addressed while interpreting the findings. Firstly, only healthy young adults were recruited in present study. So, reliability of the device to measure other clinical populations is warranted to establish reliability of the device in various pathological conditions that exhibit confounding factors like pain, deformity, and gait alterations to explore reproducibility of PP data conducted one week apart. Secondly, the device is unable to capture consecutive steps and is designed to capture one step at a time.

\section{CONCLUSION}

The results firmly establish that OHM 3000 is a reliable instrument for measuring PP in static as well as dynamic conditions in healthy individuals. The device yielded 0.87 to 0.94 intrasession and intersession reliability for SPP variables: $M P, A P, W D, C A$ and exhibited 0.61 to 0.98 intrasession and intersession reliability for DPP variables: $M P, A P$, and $C A$. Overall, OHM 3000 PP system reported higher reliability similar to other commercially available pressure devices thus establishing it as an acceptable tool in clinical practice and research settings.

\section{ABBREVIATIONS}

ADL- Activities of daily living,

GRF- Ground reaction force,

COP- Center of pressure,

PP- Plantar pressure,

FPI-6- Foot posture index-6,

SPP- static plantar pressure,

DPP- Dynamic plantar pressure,

kPa- kilopascals,

MP- Maximum pressure,

AP- Average pressure, .

WD- Weight distribution,

CA- Contact area,

SD-Standard deviation,

ICC- Interclass correlation coefficient,

CoV- Co-efficient of variances.

\section{Conflicts of interest:}

The author(s) declared no potential conflicts of interest concerning the research, authorship, and/or publication of this article.

\section{Author Contributions}

NS - Conception and design of the study.

NS and SV - Acquisition of data.

NS - Analysis and interpretation of data.

NS and SV - Drafting and final approval of the version to be submitted and published

Dr. Neha Sawant: 0000-0003-3492-9865

Dr. Sanket Vaidya: 0000-0002-2674-4564

\section{ACKNOWLEDGEMENTS}

We are thankful to our research participants, without their participation, this study would have not been possible. We also appreciate our colleagues who provided their expertise that greatly assisted the research.

\section{REFERENCES}

[1]. Manganaro D, Dollinger B, Nezwek TA, Sadiq NM. Anatomy, Bony Pelvis and Lower Limb, Foot Joints. StatPearls [Internet]. 2019 Aug 18.

[2]. Abu-Naser SS, MAHDI AO. A proposed expert system for foot diseases diagnosis. American Journal of Innovative Research and Applied Sciences 2016;2(4):155-168.

[3]. Milner CE. Functional Anatomy for Sport and Exercise: A Quick A-to-Z Reference. Routledge; 2019 Apr 25.

[4]. Brockett CL, Chapman GJ. Biomechanics of the ankle. Orthopaedics and trauma. 2016 Jun 1;30(3):232-8.

[5]. Wright WG, Ivanenko YP, Gurfinkel VS. Foot anatomy specialization for postural sensation and control. Journal of neurophysiology. 2012 Mar 1;107(5):1513-21.

[6]. Gates LS, Arden NK, Hannan MT, Roddy E, Gill TK, Hill CL et al. Prevalence of foot pain across an international Consortium of Population Based cohorts. Arthritis care \& research. 2019 May;71(5):661-70.

[7]. Hong CY, Guo LY, Song R, Nagurka ML, Sung JL, Yen $\mathrm{CW}$. Assessing postural stability via the correlation patterns of vertical ground reaction force components. Biomedical engineering online. 2016 Dec;15(1):1-8.

[8]. Vaverka F, Elfmark M, Svoboda Z, Janura M. System of gait analysis based on ground reaction force assessment. Acta Gymnica. 2015 Dec 31;45(4):187-93.

[9]. Ismail SI, Nunome H, Marzuki FF, Suaidi I. The influence of additional surface on force platforms ground reaction force data during walking and running. American Journal of Sports Science. 2018;6(3):78-82.

[10]. Wannop JW, Worobets JT, Stefanyshyn DJ. Normalization of ground reaction forces, joint moments, and free moments in human locomotion. Journal of applied biomechanics. 2012 Dec 1;28(6):665-76. 
[11]. Hills AP, Hennig EM, McDonald M, Bar-Or O. Plantar pressure differences between obese and nonobese adults: a biomechanical analysis. International journal of obesity. 2001 Nov;25(11):16749.

[12]. Hofmann UK, Götze M, Wiesenreiter K, Müller O, Wünschel M, Mittag F. Transfer of plantar pressure from the medial to the central forefoot in patients with hallux valgus. BMC musculoskeletal disorders. 2019 Dec;20(1):1-8.

[13]. Rao S, Riskowski JL, Hannan MT. Musculoskeletal conditions of the foot and ankle: assessments and treatment options. Best Practice \& Research Clinical Rheumatology. 2012 Jun 1;26(3):345-68.

[14]. Choi K, Park JH, Cheong HK. Prevalence of musculoskeletal symptoms related with activities of daily living and contributing factors in Korean adults. Journal of Preventive Medicine and Public Health. 2013 Jan;46(1):39.

[15]. Wafai L, Zayegh A, Woulfe J, Aziz SM, Begg R. Identification of foot pathologies based on plantar pressure asymmetry. Sensors. 2015 Aug;15(8):20392 408.

[16]. Bus SA, Van Deursen RW, Armstrong DG, Lewis JE, Caravaggi CF, Cavanagh PR, International Working Group on the Diabetic Foot (IWGDF). Footwear and offloading interventions to prevent and heal foot ulcers and reduce plantar pressure in patients with diabetes: a systematic review. Diabetes/metabolism research and reviews. 2016 Jan;32:99-118.

[17]. Nouman M, Leelasamran W, Chatpun S. Effectiveness of total contact orthosis for plantar pressure redistribution in neuropathic diabetic patients during different walking activities. Foot $\&$ ankle international. 2017 Aug;38(8):901-8

[18]. Izquierdo-Renau M, Pérez-Soriano P, Ribas-García $V$, Queralt A. Intra and intersession repeatability and reliability of the S-Plate ${ }^{\circledR}$ pressure platform. Gait \& posture. 2017 Feb 1;52:224-6.

[19]. McLaughlin P, Vaughan B, Shanahan J, Martin J, Linger $G$. Inexperienced examiners and the foot posture index: a reliability study. Manual therapy. 2016 Dec 1;26:238-40
[20]. AL-Baghdadi JA, Chong AK, Milburn PD. Fabrication and testing of a low-cost foot pressure sensing system. InProceedings of the 3rd International Conference on Industrial Application Engineering 2015 Mar 28 (pp. 1-9).

[21]. Franco PS, Moro CF, Figueiredo MM, Azevedo RR, Ceccon FG, Carpes FP. Within and between-days repeatability and variability of plantar pressure measurement during walking in children, adults and older adults. Advances in Rheumatology. 2019 Jul 29;58.

[22]. Rogers A, Morrison SC, Gorst T, Paton J, Freeman J, Marsden J, Cramp MC. Repeatability of plantar pressure assessment during barefoot walking in people with stroke. Journal of Foot and Ankle Research. 2020 Dec;13(1):1-7.

[23]. McKay MJ, Baldwin JN, Ferreira P, Simic M, Vanicek N, Wojciechowski E, Mudge A, Burns J, 1000 Norms Project Consortium. Spatiotemporal and plantar pressure patterns of 1000 healthy individuals aged 3-101 years. Gait \& posture. 2017 Oct 1;58:7887.

[24].Chen T. Robust Methods for Estimating the Intraclass Correlation Coefficient and for Analyzing Recurrent Event Data (Doctoral dissertation, Harvard University).

[25]. Indrayan A, Holt MP. Concise encyclopedia of biostatistics for medical professionals. Crc Press; 2016 Nov 25.

[26]. Portney LG, Watkins MP. Foundations of clinical research: applications to practice. Upper Saddle River, NJ: Pearson/Prentice Hall; 2009.

[27]. Gurney JK, Kersting UG, Rosenbaum D. Betweenday reliability of repeated plantar pressure distribution measurements in a normal population. Gait \& posture. 2008 May 1;27(4):706-9.

[28]. Martínez-Nova A, Cuevas-García JC, Pascual-Huerta J, Sánchez-Rodríguez R. BioFoot ${ }^{\circledR}$ in-shoe system: Normal values and assessment of the reliability and repeatability. The Foot. 2007 Dec 1;17(4):190-6.

How to cite this article: Neha SAWANT, Sanket VAIDYA. Reliability of OHM 3000 Plantar Pressure System for Measurement of Plantar Pressures in Healthy Indian Population. Int J Physiother Res 2022;10(1):4095-4101. DOI: 10.16965/ijpr.2021.210 